Quim. Nova, Vol. 31, No. 4, 798-801, 2008

\title{
UMA METODOLOGIA SIMPLES E EFICIENTE PARA A CLORAÇÃO DE COMPOSTOS AROMÁTICOS ATIVADOS UTILIZANDO O ÁCIDO TRICLORO-ISOCIANÚRICO ${ }^{\#}$
}

\author{
Gabriela F. Mendonça e Marcio C. S. de Mattos* \\ Departamento de Química Orgânica, Instituto de Química, Universidade Federal do Rio de Janeiro, CP 68545, 21945-970
}

Rio de Janeiro - RJ, Brasil

Recebido em 14/1/08; aceito em 14/3/08; publicado na web em 9/4/08

\begin{abstract}
A SIMPLE AND EFFICIENT METHODOLOGY FOR CHLORINATION OF ACTIVATED AROMATIC COMPOUNDS USING TRICHLOROISOCYANURIC ACID. The chlorination of activated aromatic rings is efficiently achieved under mild conditions by reaction of aromatic compounds with trichloroisocyanuric acid in acetonitrile, at room temperature, leading to products in 60-95\% isolated yields and good regioselectivity.
\end{abstract}

Keywords: trichloroisocyanuric acid; chlorination; aromatic compounds.

\section{INTRODUÇÃO}

Halo-arenos são compostos muito úteis em química, visto serem importantes produtos e intermediários com as mais diversas aplicações. ${ }^{1}$ Embora existam várias rotas descritas na literatura para a cloração eletrofílica de anéis ativados, elas são limitadas pelo emprego de reagentes perigosos, caros ou não disponíveis no mercado nacional. ${ }^{2} \mathrm{O}$ método mais comumente utilizado para a preparação de cloro-arenos envolve o emprego de $\mathrm{Cl}_{2}$, que além de ser um gás tóxico, corrosivo e difícil de manipular, gera $\mathrm{HCl}$ como subproduto, que também é altamente nocivo. ${ }^{3}$ Nesse contexto, atualmente existe um interesse muito grande por reagentes alternativos e seguros que façam a cloração eficiente de compostos orgânicos. ${ }^{4}$

As $N$-halo-amidas (Figura 1) formam uma classe de compostos de grande interesse para a química orgânica sintética, pois são fontes de halogênios eletrofílicos (íons halênios, " $\mathrm{X}^{+}$") que podem ser empregados em diversas reações. ${ }^{5}$ Dentro dessa classe, destacam-se as $N$-halo-succinimidas (Figura 1a), que são as mais estudadas, conhecidas e comercialmente disponíveis. ${ }^{6}$ Compostos similares, onde uma das carbonilas é substituída por um grupo sulfona, são os derivados da sacarina (Figura 1b). ${ }^{7}$ Os ácidos trialoisocianúricos (Figura 1c) são reagentes análogos às $N$-halo-amidas. Apesar de serem conhecidos vários ácidos dessa família, ${ }^{8}$ o mais utilizado é o tricloro-isocianúrico (TCCA, Figura $1 \mathrm{c}, \mathrm{X}=\mathrm{Cl}$ ), visto que é facilmente encontrado em supermercados, pois é um oxidante amplamente utilizado como "cloro de piscina" e fonte de cloro empregado como desinfetante em vários produtos de limpeza (comercialmente é encontrado como "cloro estabilizado" com $99 \%$ de pureza). ${ }^{9}$ Ele é um reagente estável, de fácil manipulação, obtenção e baixo custo.

Do ponto de vista da química limpa, no que diz respeito à economia atômica, ${ }^{10}$ a utilização do TCCA também se mostra bastante vantajosa quando comparada a diversos análogos $N$-clorados ${ }^{11}$ (tais como 1,3-dicloro-5,5-dimetil-hidantoína, $N$-cloro-succinimida, $N$-cloro-sacarina e cloramina-T), pois ele é capaz de transferir até $45,5 \%$ de sua massa, um valor bem superior ao dos seus análogos (Tabela 1).

"Em memória de Helena M.C. Ferraz.

*e-mail: mmattos@iq.ufrj.br<smiles>[X]N1C(=O)CCC1=O</smiles>

(a)<smiles>[X]n1sc2ccccc2c1=O</smiles>

(b)

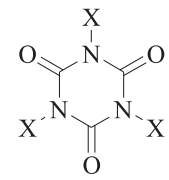

(c)

$$
\mathrm{X}=\mathrm{Cl}, \mathrm{Br}, \mathrm{I}
$$

Figura 1. Estruturas de algumas N-halo-amidas utilizadas como fontes de íons halênio em síntese orgânica: (a) N-halo-succinimidas, (b) N-haloftalimidas e (c) ácidos trialo-isocianúricos

Recentemente, nosso grupo relatou a grande eficiência do TCCA como fonte de cloro eletrofílico em reações de co-halogenação de alquenos na presença de vários nucleófilos oxigenados para gerar cloroidrinas, ${ }^{12} \beta$-cloro-éteres, ${ }^{12} \beta$-cloro-acetatos ${ }^{12}$ e $\beta$-cloroformiatos. ${ }^{13}$ Já a co-halogenação de alquenos em presença de água seguida de adição de base fornece epóxidos em altos rendimentos, ${ }^{14}$ enquanto que a co-halogenação em presença de $\mathrm{NaCl}$ se mostrou uma metodologia eficiente, limpa e bastante segura para a preparação de dicloro-alcanos vicinais. ${ }^{15}$

Embora o TCCA seja um reagente bastante utilizado em diversas transformações orgânicas, são raros os exemplos que envolvem a cloração de anéis aromáticos; estes, por sua vez, incluem reações radicalares, catalisadas por ácidos ou líquidos iônicos. ${ }^{16}$ Desta forma, dando continuidade ao nosso interesse na utilização do ácido tricloro-isocianúrico como fonte de cloro eletrofílico, o presente trabalho tem como objetivo estender essa nova metodologia para a cloração direta de compostos aromáticos ativados.

\section{RESULTADOS E DISCUSSÃO}

Os resultados da cloração de diversos anéis aromáticos ricos em elétrons com TCCA em acetonitrila encontram-se resumidos na Tabela 2. As reações foram feitas agitando-se $2 \mathrm{mmol}$ do substrato aromático com quantidade variada de ácido tricloro-isocianúrico em $10 \mathrm{~mL}$ de acetonitrila, à temperatura ambiente, sendo os produtos isolados em 60-95\% de rendimento. As reações envolvendo 
Tabela 1. Economia atômica de vários $N$-cloro compostos

$\mathrm{N}$-cloro composto

Conteúdo de cloro ativo $(\%)^{\mathrm{a}}$<smiles>O=c1n(Cl)c(=O)n(Cl)c(=O)n1Cl</smiles><smiles>CC1(C)C(=O)N(Cl)C(=O)N1Cl</smiles><smiles>O=c1c2ccccc2on1Cl</smiles><smiles>Cc1ccc([N+](=O)[O-])cc1</smiles>

${ }^{a}$ Massa do reagente incorporada ao produto.

arenos fortemente ativados para uma substituição eletrofílica aromática (2-metóxi-naftaleno, 1,4-dimetóxi-benzeno, anisol) ocorreram num curto período de tempo (0,2-1,5 h), utilizando-se quantidade estequiométrica de TCCA (0,34 equiv. mol). Por outro lado, nas reações envolvendo arenos fracamente ativados (acetanilida, naftaleno, tolueno), houve a necessidade de utilização de um pequeno excesso de reagente ( 0,5 equiv. mol), para acelerar a reação (Tabela 2, entradas $4,5,8,9$ ), sendo que, no caso do tolueno, a conversão não foi completa. Como era de se esperar, arenos não ativados (benzeno e cloro-benzeno) não reagiram, mesmo após 5 dias.

No geral, os produtos apresentaram alta regiosseletividade. Todavia, em alguns casos foi verificada uma mistura de produtos clorados nas posições orto e para em diferentes proporções (Tabela 2, entradas 6-9). A reação da 4,4'-dimetóxi-bifenila forneceu uma mistura de produtos mono- e diclorados ao se utilizar 0,34 equiv. mol de TCCA. Dessa forma, optou-se pela utilização de 0,67 equiv. mol de TCCA, obtendo-se assim apenas o produto diclorado em $91 \%$ de rendimento após 5 h de reação.
Na cloração do dureno com TCCA ( 0,5 equiv. mol), com apenas 10 min de reação, o substrato já havia sido totalmente consumido. Curiosamente, além de ter sido observada predominantemente a incorporação de cloro no anel (reação eletrofílica de monoe dicloração), observou-se também a incorporação de cloro no grupo metila, o que caracteriza uma reação radicalar (Esquema 1). Esse mesmo comportamento foi observado na reação do dureno com a $\mathrm{N}$-cloro-sacarina. ${ }^{22}$

\section{CONCLUSÕES}

Concluindo, esta nova metodologia sintética proposta para cloração de compostos aromáticos ativados mostrou-se bastante eficiente e mais adequada que as tradicionais (reações com cloro, NCS, etc.). O reagente utilizado (TCCA) é seguro, barato e disponível no mercado nacional, além das reações serem simples, ocorrerem em condições muito brandas (temperatura ambiente, curto período de tempo) e levando a altos rendimentos.

\section{PARTE EXPERIMENTAL}

\section{Geral}

O ácido tricloro-isocianúrico (comercial, 98\%), todos os arenos e solventes foram usados como recebidos. As análises cromatográficas (CGAR) foram realizadas em um cromatógrafo a gás HP-5890-II com FID e coluna capilar de sílica fundida RTX-5 com 30 m de comprimento, 0,25 mm de diâmetro interno e $0,5 \mu \mathrm{m}$ de espessura de fase. As análises de CGAR-EM foram realizadas em um cromatógrafo a gás HP-6890 acoplado a um detector seletivo de massas HP-5973 com coluna capilar de sílica fundida HP-5, $30 \mathrm{~m}$ de comprimento, 0,25 $\mathrm{mm}$ de diâmetro interno e $0,25 \mu \mathrm{m}$ de espessura de fase.

\section{Procedimeto geral para a cloração de arenos ativados com TCCA}

A uma solução do composto aromático $(2 \mathrm{mmol})$ em acetonitrila $(10 \mathrm{~mL})$, adicionou-se ácido tricloro-isocianúrico $(0,67-1,34 \mathrm{mmol}$ - ver Tabela 2) à temperatura ambiente e sob agitação. Após o término da reação (constatado através do desaparecimento da coloração azulada ao se pingar uma alíquota da mistura reacional em um papel embebido com solução de KI amidonado), filtrou-se o ácido cianúrico (sólido branco que precipita com o decorrer da reação) e o líquido foi extraído (AcOEt) e lavado com sol. 15\% de $\mathrm{NaHSO}_{3}$ e com água. Após secagem $\left(\mathrm{Na}_{2} \mathrm{SO}_{4}\right)$ e evaporação do solvente, os cloro-arenos foram caracterizados por co-injeção com padrão na CGAR, ponto de fusão e espectrometria de massas.

\section{Dados selecionados}

1-cloro-2-metóxi-naftaleno

P.F. $69-70,5{ }^{\circ} \mathrm{C}$ (lit. $\left.{ }^{17} 68-70{ }^{\circ} \mathrm{C}\right)$. E.M. $(70 \mathrm{eV}): \mathrm{m} / \mathrm{z}$ 99, 114, 126, 149 (100\%), 151, 177, 179, $192\left(\mathrm{M}^{+}\right), 194\left(\mathrm{M}^{+}+2\right)$.<smiles>Cc1cc(C)c(C)cc1C</smiles>

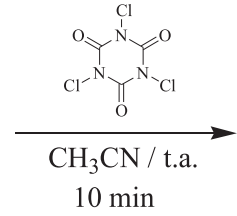<smiles>Cc1cc(C)c(CCl)cc1C</smiles>

$(1: 0,4: 0,15)$<smiles>Cc1c(C)c(Cl)c(C)c(C)c1Cl</smiles> 
Tabela 2. Resultados obtidos na cloração de arenos com TCCA

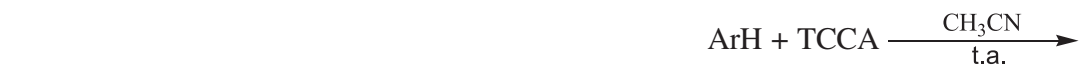

\begin{tabular}{lllll}
\hline Entrada Substrato & $\mathrm{t}(\mathrm{h})$ & Rend. $(\%)^{\mathrm{a}}$ & Ref. \\
\hline $1^{\mathrm{b}}$ & 0,2 & 95 & 17
\end{tabular}

$2^{\mathrm{b}}$<smiles>COc1ccc(OC)cc1</smiles>

$3^{\mathrm{b}}$<smiles>COc1ccc(Br)cc1</smiles>

$4^{c}$<smiles>c1ccc2ccccc2c1</smiles>

$5^{c}$<smiles>c1ccc2c(c1)oc1ccccc12</smiles>

$6^{\mathrm{b}}$<smiles>COc1ccccc1</smiles><smiles>CC(=O)N(C)c1ccccc1</smiles>

$8^{c}$<smiles>CC(=O)Nc1ccccc1</smiles>

$9^{c}$<smiles>Cc1ccccc1</smiles>

$10^{\mathrm{e}}$<smiles>COc1ccc(-c2ccc(OC)cc2)cc1</smiles>

$11^{\mathrm{c}}$<smiles>Clc1ccccc1</smiles>

$12^{\mathrm{c}}$<smiles>COc1ccc(OC)c(Cl)c1</smiles><smiles>COc1ccc(Br)cc1Cl</smiles><smiles>Clc1cccc2ccccc12</smiles><smiles>Clc1ccc2oc3ccccc3c2c1</smiles><smiles>COc1ccc(Cl)cc1</smiles>

$(1: 5)^{d}$<smiles>CC(=O)N(C(C)=O)c1ccc(Cl)cc1</smiles>

$(1: 3)^{d}$<smiles>CC(=O)Nc1ccc(Cl)cc1</smiles>

$(1: 3)^{\mathrm{d}}$<smiles>Cc1ccc(Cl)cc1</smiles>

$(1: 1)^{\mathrm{d}}$<smiles>COc1ccc(-c2ccc(OC)c(Cl)c2)cc1Cl</smiles>

não reage

não reage 
4-bromo-2-cloro-anisol

P.F. $68-69{ }^{\circ} \mathrm{C}$ (lit. $\left.{ }^{19} 68-70{ }^{\circ} \mathrm{C}\right)$. E.M. $(70 \mathrm{eV}): \mathrm{m} / \mathrm{z}$ 177, 179, 181, 205, 207, 209, $220\left(\mathrm{M}^{+}\right), 222\left(\mathrm{M}^{+}+2\right), 224\left(\mathrm{M}^{+}+4\right)$.

\section{3,3'-dicloro-4,4'-dimetóxi-bifenila}

P.F. $156,5-159{ }^{\circ} \mathrm{C}$ (lit. $\left.{ }^{24} 156-158{ }^{\circ} \mathrm{C}\right)$. E.M. $(70 \mathrm{eV}): \mathrm{m} / \mathrm{z}, 125$, 207, 224, 239, 267, 269, $282\left(\mathrm{M}^{+}\right), 284\left(\mathrm{M}^{+}+2\right), 286\left(\mathrm{M}^{+}+4\right)$.

\section{2-cloro-naftaleno}

EM (70 eV): $m / z, 101,126,127,162\left(\mathrm{M}^{+}, 100 \%\right), 164\left(\mathrm{M}^{+}+2\right)$.

4-cloro-dibenzofurano

EM (70 eV): $\mathrm{m} / \mathrm{z} 101,126,127,162\left(\mathrm{M}^{+}, 100 \%\right), 164\left(\mathrm{M}^{+}+2\right)$.

2-cloro-1,4-dimetóxi-benzeno

EM (70 eV): $m / z, 79,107,129,131,157$ (100\%), 159, $172\left(\mathrm{M}^{+}\right)$, $174\left(\mathrm{M}^{+}+2\right)$

\section{AGRADECIMENTOS}

Agradecemos ao CNPq pelas bolsas de doutorado (G. F. Mendonça) e de pesquisa (M. C. S. de Mattos).

\section{REFERENNIAS E NOTAS}

1. Groger, H.; J. Prakt. Chem. 2000, 342, 334

2. De La Mare, P. B.; Electrophilic Halogenation, Cambridge University Press: Cambridge, 1976, cap. 5 e 7; Larock, R. C.; Comprehensive Organic Transformation: A Guide to Functional Group Preparation, $2^{\text {nd }}$ ed., WileyVCH: Nova York, 1999.

3. The Merk Index, $13^{\text {th }}$ ed., Merck \& Co. Inc.: Whitehouse Station, 2001.

4. Zhang, Y.; Shibatomi, K.; Yamamoto, H.; Synlett 2005, 2837; Dewkar, G. K.; Narina, S. V.; Sudalai, A.; Org. Lett. 2003, 5, 4501.

5. Para uma excelente revisão recente sobre a utilização de $N$-halo compostos em síntese orgânica veja: Kolvani, E.; Ghorbani-Choghamarani, A.; Salehi, P.; Shirini, F.; Zolfigol, M. A.; J. Iran. Chem. Soc. 2007, 4, 126.

6. Para uma revisão sobre $N$-halo-succinimidas veja: Koval', I. V.; Russ. J. Org. Chem. 2002, 38, 301
7. Para uma revisão sobre $N$-halo-sacarinas veja: de Souza, S. P .L.; da Silva, J. F. M.; de Mattos, M. C. S.; Quim. Nova 2006, 29, 1061.

8. Ácido tribromo-isocianúrico: de Almeida, L. S; Esteves, P. M.; de Mattos, M. C. S.; Synthesis 2006, 221; ácido bromo-dicloro-isocianúrico: de Almeida, L. S.; Esteves, P. M.; de Mattos, M. C. S.; Synlett 2007, 1687; ácido triiodo-isocianúrico: Ribeiro, R. da S.; Esteves, P. M.; de Mattos, M. C. S.; Tetrahedron Lett. 2007, 48, 8747

9. Para revisões sobre TCCA veja: Tilstan, U.; Weinmann, H.; Org. Process Res. Dev. 2002, 6, 384; Barros, J. C.; Synlett 2005, 2115; Cunha, A. C.; Paixão, F. M.; Souza, M. C. B. V.; Ferreira, V. F.; Quim. Nova 2006, 29, 520; Blotny, G.; Tetrahedron 2006, 62, 9507.

10. Sanseverino, A. M.; Quim Nova 2000, 23, 102; Lenardão, E. J.; Freitag, R. A.; Dabdoub, M. J.; Batista, A. C. F.; Silveira, C. C.; Quim Nova 2003 , 26, 123; Anastas, P. T.; Warner, J. C.; Green Chemistry: Theory and Practice, Oxford University Press: Oxford, 2000.

11. Kumar, V.; Kaushik, M. P.; Synlett 2007, 2937.

12. Mendonça, G. F.; Sanseverino, A. M.; de Mattos, M. C. S.; Synthesis 2003, 45 .

13. de Souza, A. V. A.; Mendonça, G. F.; Bernini, R. B.; de Mattos, M. C. S.; J. Braz. Chem. Soc. 2007, 18, 1575.

14. Wengert, M.; Sanseverino, A. M.; de Mattos, M. C. S.; J. Braz. Chem. Soc. 2002, 13, 700.

15. Tozetti, S. D. F.; de Almeida, L. S.; Esteves, P. M.; de Mattos, M. C. S.; J. Braz. Chem. Soc. 2007, 18, 675.

16. Mendonça, G. F.; Magalhães, R. R.; Esteves, P. M.; de Mattos, M. C. S.; J. Braz. Chem. Soc. 2005, 16, 695; Juenge, E. C.; Beal, D. A.; Duncan, W. P.; J. Org. Chem. 1970, 35, 719; Rosevear, J.; Wilshire, J. F. K.; Austr. J. Chem. 1980, 33, 843; Hubbard, A.; Okazaki, T.; Laali, K. K.; Austr. J. Chem. 2007, 60, 923; Bastos, R. S.; Cunha, A. S.; Silva, L. C.; Oliveira, C. C. P.; Rezende C. M.; Pinto, A. C.; Quim. Nova 2008, 31, 172.

17. Baumgartner, M. T.; Tempesti, T. C.; Pierini, A. B.; ARKIVOC 2003, 420.

18. Barhate, N. B.; Gajare, A. S.; Wakharkar, R. D.; Bedekar, A. V.; Tetrahedron 1999, 55, 11127.

19. Koo, B. -S.; Kim, E. -H.; Lee, K. -J.; Synth. Commun. 2002, 32, 2275.

20. Lo, C. -Y.; Kumar, M. P.; Chang, H. -K.; Lush, S. -F.; Liu, R. -S.; J. Org. Chem. 2005, 70, 10482.

21. Wassmundt, F. W.; Pedemonte, R. P.; J. Org. Chem. 1995, 60, 4991.

22. de Souza, S. P. L.; da Silva, J. F. M.; de Mattos, M. C. S.; J. Braz. Chem. Soc. 2003, 14, 832

23. Newkome, G. R.; Xia, Y. J.; Heterocycles 1983, 20, 981; Bunnett, J. F.; Kato, T.; Flynn, R. R.; Skorcz, J. A.; J. Org. Chem. 1963, 28, 1.

24. Bovicelli, P.; Antonioletti, R.; Onori, A.; Delogu, G.; Fabbri, D.; Dettori, M. A.; Tetrahedron 2006, 62, 635. 